\title{
EVALUATION OF INHOMOGENEOUS RESISTIVE LAYERS BY A FOUR POINT METHOD
}

\author{
GYÖRGY FODOR, MIKLÓS SZILÁGYI and LÁSZLÓ ZOMBORY \\ Technical University of Budapest, H-1521 Budapest, Hungary
}

(Received August 1, 1979; in final form May 17, 1980)

\begin{abstract}
This paper describes the determination of the sheet resistance of a narrow strip located in a homogeneous resistive layer of different resistivity. The application of the method for the evaluation of inhomogeneous layers used for thermoprinter head production is discussed.
\end{abstract}

\section{INTRODUCTION}

The four point measurement is widely used in the electronic component industry for fabrication control. This measurement can be applied to determine for example the resistivity of semiconductor ingots, ingot parts or samples of special shape. ${ }^{1}$ Applications of this method are extremely important when it is carried out on very thin layers in order to determine of the sheet resistance (the ratio of the resistivity and the layer thickness). ${ }^{2}$

In the fundamental case of the sheet resistance measurement the following conditions must be satisfied:

- the layer thickness $w$ is far less than the uniform distance $s$ between the neighbours of the four contacts placed along a straight line.

- the linear sizes of the layer perpendicular to the thickness are far larger than the distance $s$.

- the probes are placed far from the edges of the sample.

Given these circumstances the sheet resistance $R_{\square}$ can be calculated from the current $I$ flowing between the outside contacts and the potential difference $\Delta \phi$ between the inside ones by the simple formula

$$
R_{\square}=\frac{\pi}{\ln 2} \frac{\Delta \phi}{I}
$$

The determination of the sheet resistance is often necessary in cases when several of the conditions above are not satisfied. In these cases Eq. (1) must be modified by considering correction factors. Smits ${ }^{2}$ and other authors determined the correction factors for given cases applying the method of conformal mapping or the method of images. These corrections relate to those cases where the sample sizes are comparable to the pin distance or the measurement is executed near the edges of the sample. The cases examined can be divided to two groups:

- at the edges of the sample the layer is discontinuous

- the edge of the sample is connected to conductive $\left(R_{\square}=0\right)$ layer.

The calculations till now have not related to the case where the resistance changes to a finite degree inside the layer. The present paper deals with four point measurement of this kind of layer with the special configuration shown in Figure 1.

\section{DETERMINATION OF SHEET RESISTANCE OF AN INHOMOGENEOUS STRIP}

The problem is to determine the sheet resistance of a strip different from other parts of the layer. The width of the strip is known and is less than the distance between the pins. The problem will be taken back to two sheet resistance measurements: the sheet resistance of the homogeneous layer will be measured at first far from the strip and then an apparent sheet resistance $R_{m}$ will be determined according to the geometry shown in Figure 1. 


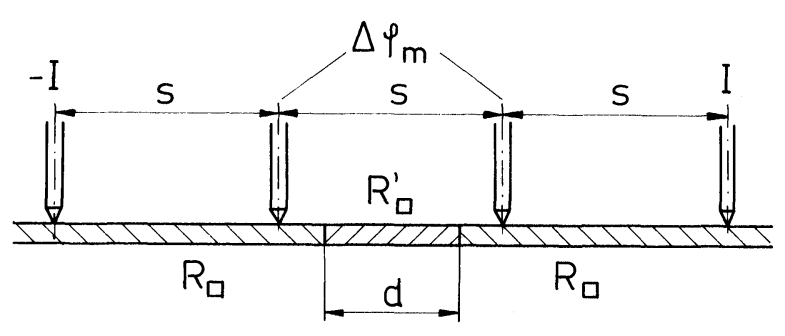

FIGURE 1 Measurement of $R_{m}$ (apparent sheet resistance).

$$
R_{m}=\frac{\pi}{\ln 2} \frac{\Delta \phi_{m}}{I}
$$

$\Delta \phi_{m}$ can be calculated applying the method of images as follows. The configuration to be calculated and the applied coordinate system can be seen in Figure $2 \mathrm{a}$. The symmetry of the configuration allows the examination of the half space $0<x<\infty$ only.

In Figure 2.b. the fictive currents are shown whose resultant field corresponds to that of the original configuration in the domain $0<x<d / 2$. In Figure 2.c. the resultant field corresponds to that of the original configuration in the domain $d / 2<x<\infty$. It is apparent that the images in both cases are situated outside of the regions where the resultant field is calculated. The resultant field of the currents $I_{0}^{*}, I_{1}^{*}$ and $+I_{1}^{\prime}$ assures the satisfaction of the boundary conditions at the plane $x=d / 2$ (generalized method of images). The current $-I_{1}^{\prime}$ assures the satisfaction of the condition $\phi(O)=0$. The resultant field of the currents $-I_{1}^{\prime}, I_{0}$ and $+I_{2}^{\prime}$ satisfies the boundary conditions at the plane $x=d / 2$. The current $-I_{2}^{\prime}$ is the image corresponding to the plane $x=0$. The currents $I_{1}$ and $+I_{3}^{\prime}$ should be added, followed by $I_{3}^{\prime}$, then $I_{2}$ and $+I_{4}^{\prime}$ etc. As a check one can see that the sum of the substituting currents is $+I$ in the domain $0<x<d / 2$ and $-I$ in the domain $d / 2<x<\infty$. Furthermore, if $R_{\square}=R_{\square}^{\prime}$, i.e. $m=1$ is valid then in both domains only the currents $+I$ and $-I$ remain corresponding to the simple images.

On the basis of the substituting configuration corresponding to the domain $0<x<d / 2$

$\phi\left(\frac{d}{2}\right)$ is given by:

$$
\phi\left(\frac{d}{2}\right)=\frac{I R_{\square}^{\prime}}{2 \pi} m \sum_{n=0}^{\infty}(1-m)^{n} \ln \frac{a+n d+d / 2}{a+n d-d / 2}
$$

and similarly in the domain $d / 2<x<\infty$

$$
\begin{aligned}
\phi(x) & =\phi\left(\frac{d}{2}\right)+\frac{I R_{\square}}{2 \pi}\left\{\ln \frac{a-d / 2}{a-x}\right. \\
& +(l-m) \ln \frac{a-d / 2}{a-d+x} \\
& \left.-(2-m) m \sum_{n=0}^{\infty}(1-m)^{n} \ln \frac{a+n d+d / 2}{a+n d+x}\right\}
\end{aligned}
$$

where

$$
m=\frac{2 R_{\square}}{R_{\square}+R_{\square}^{\prime}}
$$

Substituting $x=s / 2$ and $a=3 s / 2$ into Eq (4) gives

$$
\begin{aligned}
\frac{1}{2} \Delta \phi_{m} & =\phi\left(\frac{s}{2}\right) \\
& =\frac{I R_{\square}}{2 \pi}\left\{(2-m) \sum_{n=0}^{\infty}(1-m)^{n}\right. \\
& \times \ln \frac{3+(2 n+1) d / s}{3+(2 n-1) d / s} \\
& +\ln \frac{3-d / s}{2}+(1-m) \ln \frac{3-d / s}{4-2 d / s} \\
& -(2-m) m \sum_{n=0}^{\infty}(1-m)^{n} \\
& \left.\times \ln \frac{3+(2 n+1) d / s}{4+2 n d / s}\right\}
\end{aligned}
$$

Introducing the parameter $t=d / s$, the ratio of the sheet resistances measured in the homogeneous and inhomogeneous part of the sample is obtained:

$$
\begin{gathered}
\frac{R_{\square}}{R_{m}}=\ln 2\left\{\ln \frac{3-t}{2}-(1-m) \ln \frac{4-2 t}{3-t}\right. \\
-(2-m) \sum_{n=0}^{\infty}(1-m)^{n} \ln \frac{[3+(2 n+1) t]^{m-1}[3+(2 n-1) t]}{(4+2 n t)^{m}}
\end{gathered}
$$

The diagram in Figure 3. has been calculated on the basis of the formula above. After having measured $R_{\square}$ and $R_{m}$ the value of $m$ can be read from the diagrams knowing $t$.

Finally the value of $R_{\square}^{\prime}$ can be calculated from Eq. (5). 

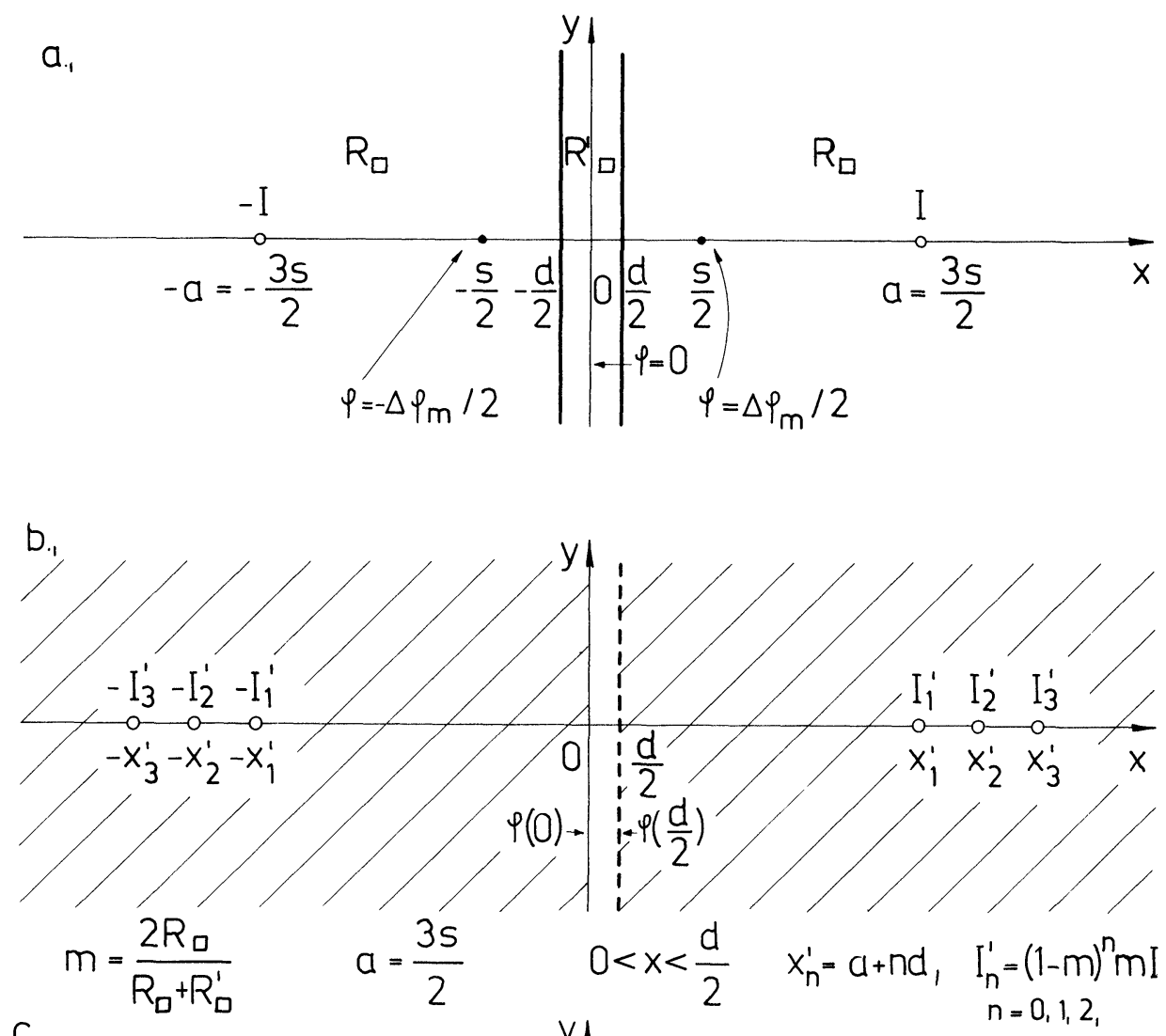

C.

y) $n=0,1,2$,

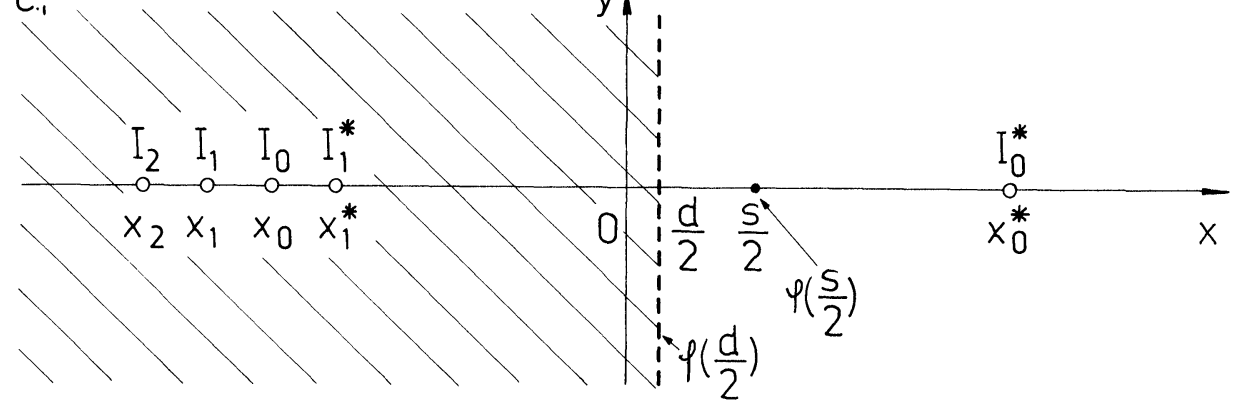

$$
\begin{aligned}
\frac{d}{2}<x<0 \quad x_{0}^{*} & =a \quad I_{0}^{*}=I \\
x_{1}^{*} & =-(a-d) \quad I_{1}^{*}=(1-m) I \\
x_{n} & =-(a+n d) \\
I_{n} & =-(1-m)^{n}(2-m) m I \\
n & =0,1,2, \ldots
\end{aligned}
$$

FIGURE 2 Generalized method of images. 


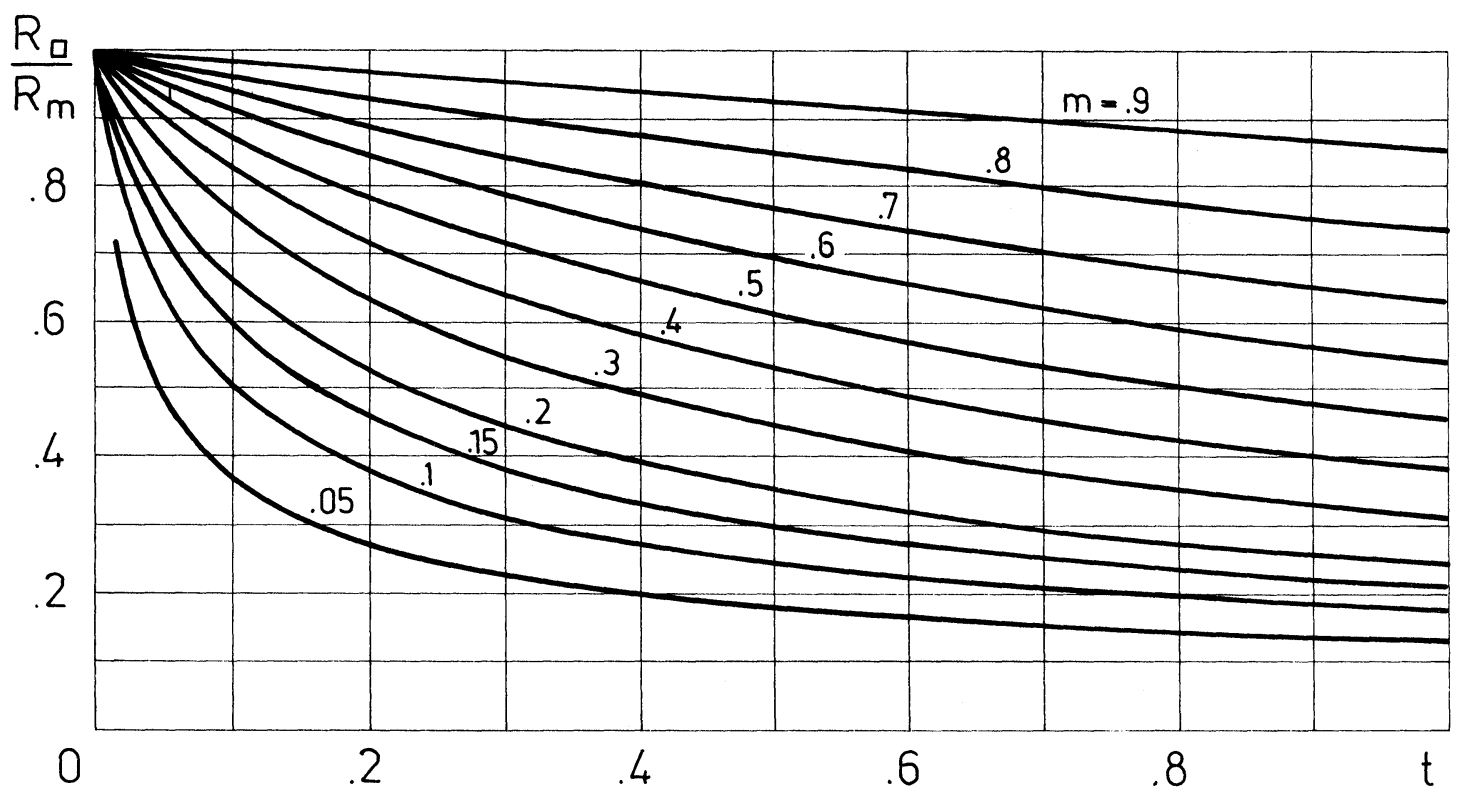

FIGURE 3 Diagrams for evaluation of sheet resistance of a strip.

It is interesting to examine a special limit case of the potential distribution $\phi(x)$ in Eq. (4). Suppose that the width $d$ of the inhomogeneous strip is very small compared to the pin distance $s$ and at the same time its sheet resistance is much higher than that of the homogeneous layer, i.e. as a limit

$$
\begin{aligned}
& d \rightarrow 0 \\
& R_{\square}^{\prime} \rightarrow \infty
\end{aligned}
$$

but the product $d . R_{\square}^{\prime}$ is positive and finite.

To calculate the limit value apply the following (but here not proved) lemma:

If the values of the positive, monotonic decreasing $f(x)$ function in the $x=0,1,2 \ldots$ distinct integer points correspond to the positive, monotonic decreasing terms of the infinite series $\left\{a_{n}\right\}$, and both the sum $\sigma=\sum_{n=0}^{\infty} a_{n}$ and the integral $\theta=\int_{0}^{\infty} f(x) \mathrm{d} x$ exist then

$$
\theta \leqslant \sigma \leqslant a_{0}+\theta
$$

is valid.

Applying Eq. (9), the sum of the series appearing in Eqs. (3) and (4) becomes

$$
\sigma=\sum_{n=0}^{\infty}(1-m)^{n} \ln \frac{a+n d+d / 2}{a+n d+\xi}
$$

where $\xi=-d / 2$ in Eq. (3) and $\xi=x$ in Eq. (4) can be estimated by the integral

$\theta=\int_{0}^{\infty} \mathrm{e}^{-\mu x} \ln x+\frac{a}{d}+\frac{1}{2}-\ln \left(x+\frac{a}{d}+\frac{\xi}{d}\right) \mathrm{d} x$

where

$$
\mu=-\ln (1-m)=2\left[\frac{R_{\square}}{R_{\square}^{\prime}}+\frac{1}{3}\left(\frac{R_{\square}}{R_{\square}^{\prime}}\right)^{3}+\ldots\right]
$$

The integral above is easy to calculate:

$$
\begin{aligned}
\theta=\frac{1}{\mu}\left[\ln \frac{a+d / 2}{a+\xi}\right. & -\exp \left(\mu \frac{a+d / 2}{d}\right) \operatorname{Ei}\left(-\mu \frac{a+d / 2}{d}\right) \\
+ & \left.\exp \left(\mu \frac{a+\xi}{d}\right) \operatorname{Ei}\left(-\mu \frac{a+\xi}{d}\right)\right]
\end{aligned}
$$

where $\operatorname{Ei}(-x)=-\int_{-x}^{\infty} \exp (-x) / x \mathrm{~d} x(x>0)$ is the exponential integral. Substituting $\xi=d / 2$ into Eq. (12) one obtains on the basis of Eq. (9) and (3)

$$
\begin{aligned}
\phi\left(\frac{d}{2}\right) & =\frac{I R_{\square}^{\prime}}{2 \pi}\left[\ln \frac{a+d / 2}{a-d / 2}-\exp \left(2 \frac{R_{\square}}{R_{\square}^{\prime}} \frac{a+d / 2}{d}\right)\right. \\
& \times \operatorname{Ei}\left(-2 \frac{R_{\square}}{R_{\square}^{\prime}} \frac{a+d / 2}{d}\right) \\
& \left.+\exp \left(2 \frac{R_{\square}}{R_{\square}^{\prime}} \frac{a-d / 2}{d}\right) \operatorname{Ei}\left(-2 \frac{R_{\square}}{R_{\square}^{\prime}} \frac{a-d / 2}{d}\right)\right] \\
& +\mathrm{O}\left(\frac{R_{\square}}{R_{\square}^{\prime}}\right)+\mathrm{O}\left(\frac{d}{a}\right)
\end{aligned}
$$


It can be seen that both the series and the integral are uniformly convergent with respect to $m$ and $d$ if $R_{\square} / R_{\square}^{\prime} \geqslant \epsilon>0$ and $d / a \geqslant \delta>0$ are valid.

Consequently the limit value of the sum and that of the integral can be calculated in the case $d \rightarrow+0$, $1 / R_{\square}^{\prime} \rightarrow+0$, and the two limit values are equal, because $\lim a_{0}=0$. Executing the limit calculation the result is

$$
\phi(+0)=-\frac{I R_{\square}}{\pi} \exp \left(2 \frac{a R_{\square}}{d R_{\square}^{\prime}}\right) \operatorname{Ei}\left(-2 \frac{a R_{\square}}{d R_{\square}^{\prime}}\right)
$$

Applying Eq. (4) and the value of $\phi(+0)$ instead of $\phi(d / 2)$ the potential distribution $\phi(x)$ along the $x$ axis can be obtained on a similar way:

$$
\begin{aligned}
\phi(x) & =\frac{I R_{\square}}{2 \pi}\left\{\ln \frac{a+x}{|a-x|}-2 \exp \frac{2(a+x)}{d R_{\square}^{\prime}}\right. \\
& \left.\times \operatorname{Ei}\left[-\frac{2(a+x) R_{\square}}{d R_{\square}^{\prime}}\right]\right\}
\end{aligned}
$$

Making the substitutions $x=s / 2, a=3 s / 2$ corresponding to the pin configuration and introducing the parameter

$$
\mathrm{Z}=\frac{4 s R_{\square}}{d R_{\square}^{\prime}}
$$

gives (similarly to Eq. (7)

$$
\frac{R_{\square}}{R_{m}}=\frac{1}{1-\frac{2}{\ln 2} \exp (Z) \operatorname{Ei}(-Z)}
$$

The diagram of Eq. (17) can be seen in Figure 4. After having measured both $R_{\square}$ and $R_{m}$ the parameter $\mathrm{Z}$ can be determined from the diagram and furthermore the product $d R_{\square}^{\prime}$ can be obtained from Eq. (16).

The relative error of $R_{\square}^{\prime}$ calculated from Eq. (17) via Eq. (16) can be estimated by determining the values of $Z$ from Figure 4, belonging to a value of $R_{\square} / R_{m}$ and either to the limiting case $(t \rightarrow 0): Z_{0}$ or to the measured $t: Z_{t}$. Supposing that all the other parameters are exact, the relative error has the following form:

$$
\frac{\Delta R_{\square}^{\prime}}{R_{\square}^{\prime}}=\frac{R_{\square}^{\prime}\left(Z_{0}\right)-R_{\square}^{\prime}\left(Z_{t}\right)}{R_{\square}^{\prime}\left(Z_{t}\right)}=\frac{Z_{t}}{Z_{0}}-1
$$

It can be seen from Figure 4. that the absolute value of the relative error is less than $10 \%$ if $t \leqslant 0,4$ and $R_{\square} / R_{m} \leqslant 0,5$.

\section{APPLICATION AND MEASUREMENT EXPERIENCES}

The method shown above seems to be applicable in several areas of electronic component manufacture, although it has been developed essentially for the purposes of production testing of inhomogeneous thin film thermoprinter heads. ${ }^{3}$

By thinning down the resistive layer at thermal points, i.e. increasing locally the sheet resistance, a thicker layer of lower resistance can be used outside

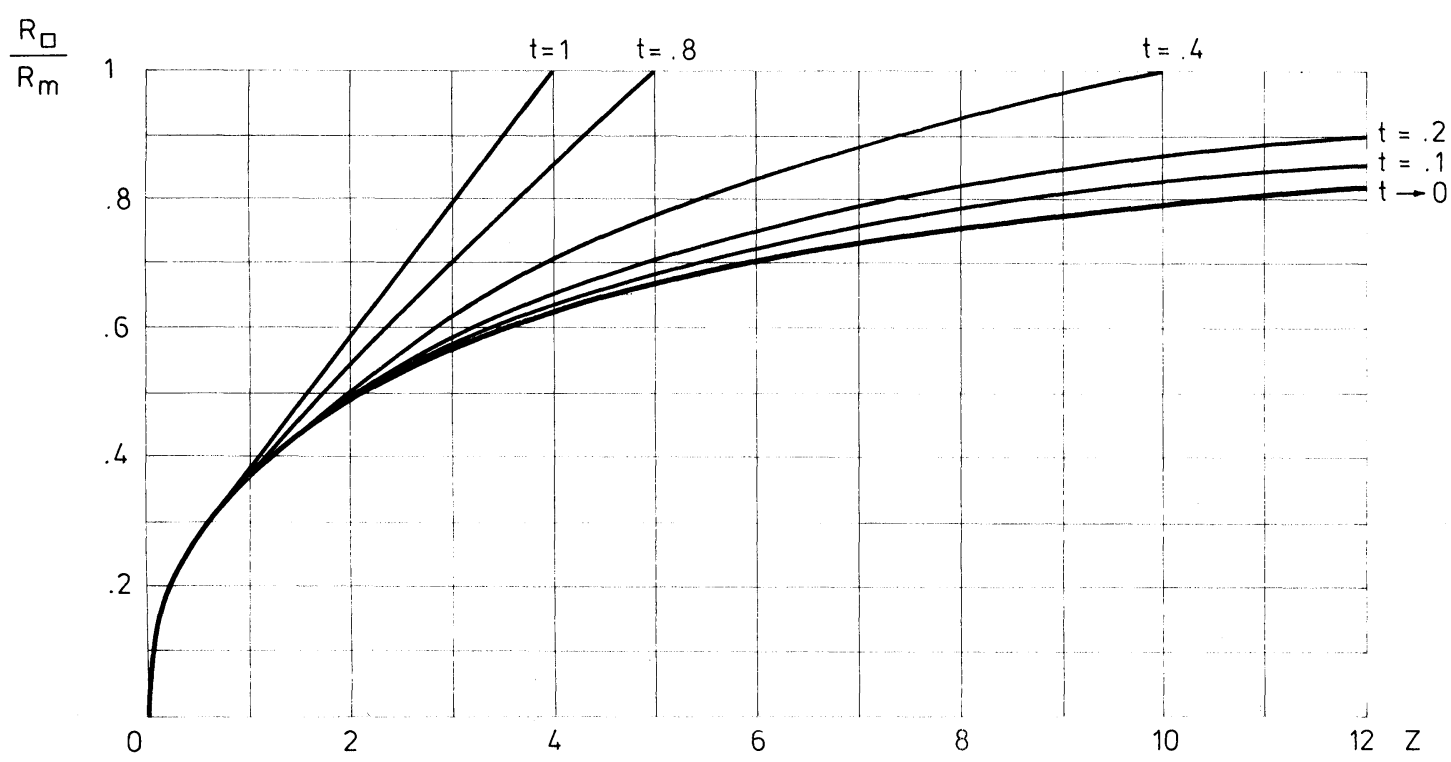

FIGURE 4 Diagrams for evaluation of sheet resistance of a narrow strip. 


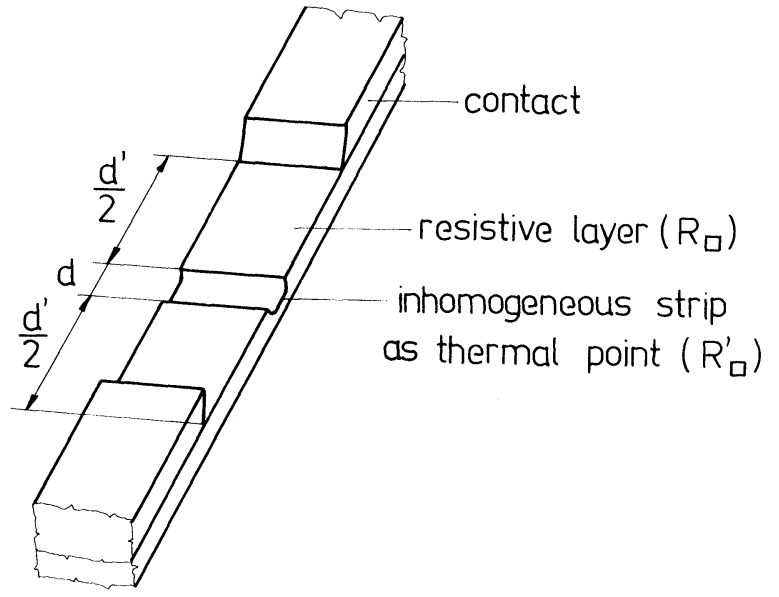

FIGURE 5 Construction of inhomogeneous thin film thermal point.

of thermal points making it possible to begin the contact layer, produced usually from a softer material, farther from the thermal point (Figure 5.). Therefore the thermo-sensitive paper does not wear down the contact layer.

An important constructional viewpoint for thermoprinter heads of this kind of configuration is that the dissipation in the layer placed from the contact to the inhomogeneous strip should be lower than that in the thermal point, i.e.

$$
d^{\prime} . R_{\square}<d . R_{\square}^{\prime}
$$

A microscopic or stylus method ${ }^{4}$ can be used to determine $d$. However the exact determination of $d$ is rather difficult because the transition between the two parts of different sheet resistivity is not sharp. As far as the approximation of Eq. (8) can be applied the product $d . R_{\square}^{\prime}$ can be determined directly by the measurement made on the resistive layer for thermoprinter heads and using Eq. (17) with Figure 4.

The typical measured values were the following: $s=1 \mathrm{~mm}, d \cong 0.2 \mathrm{~mm}, R_{\square}=20 \Omega$. The measurement resulted in about 0.5 for the value of $R_{\square} / R_{m}$. Thus from Figure $3, Z \cong 2, d R_{\square}^{\prime}=40 \Omega \mathrm{mm}$ and finally $R_{\square}^{\prime}=200 \Omega$. Determining the value of $R_{\square}^{\prime}$ using Eq. (7) and Figure 3., gave a result corresponding to that previously obtained (inside measurement errors.) This has been expected on the basis of computer comparison of Eqs. (7) and (17).

A further result is that the measured value of $R_{m}$ changes negligibly with the position of the inhomogeneous strip between the voltage measuring pins. This can be supported theoretically by examining the expression of Eq. (4): the value of the potential changes slightly in the surroundings of $x=d / 2$.

\section{REFERENCES}

1. L. B. Valdes, "Resistivity measurements on germanium for transistors" Proc. I.R.E. 42, 420 (1954).

2. F. M. Smits, "Measurement of sheet resistivities with the four point probe" J. Bell Syst. Techn. 37, 711 (1958).

3. M. Szilágyi, "Production, measurement and application of thin inhomogeneous films" Proc. Seminar "Test method in Electronics Technology"' (Balatonfüred, 1979) p. 72.

4. G. Breitweiser, "Surface profile measurements - a survey of application in the area of vacuum deposition" J. Vacuum Sci. Technol. 11, 101 (1974). 

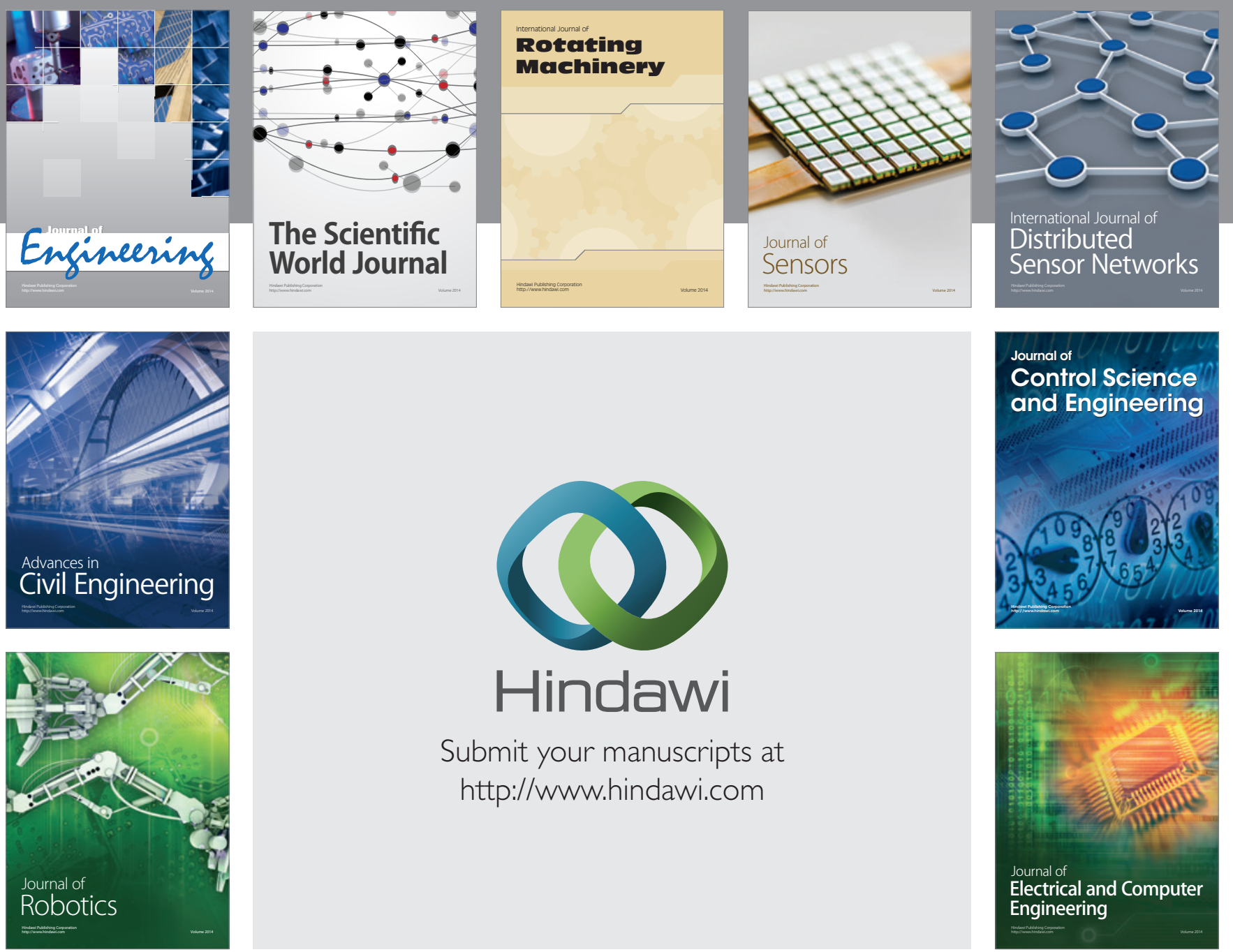

Submit your manuscripts at

http://www.hindawi.com
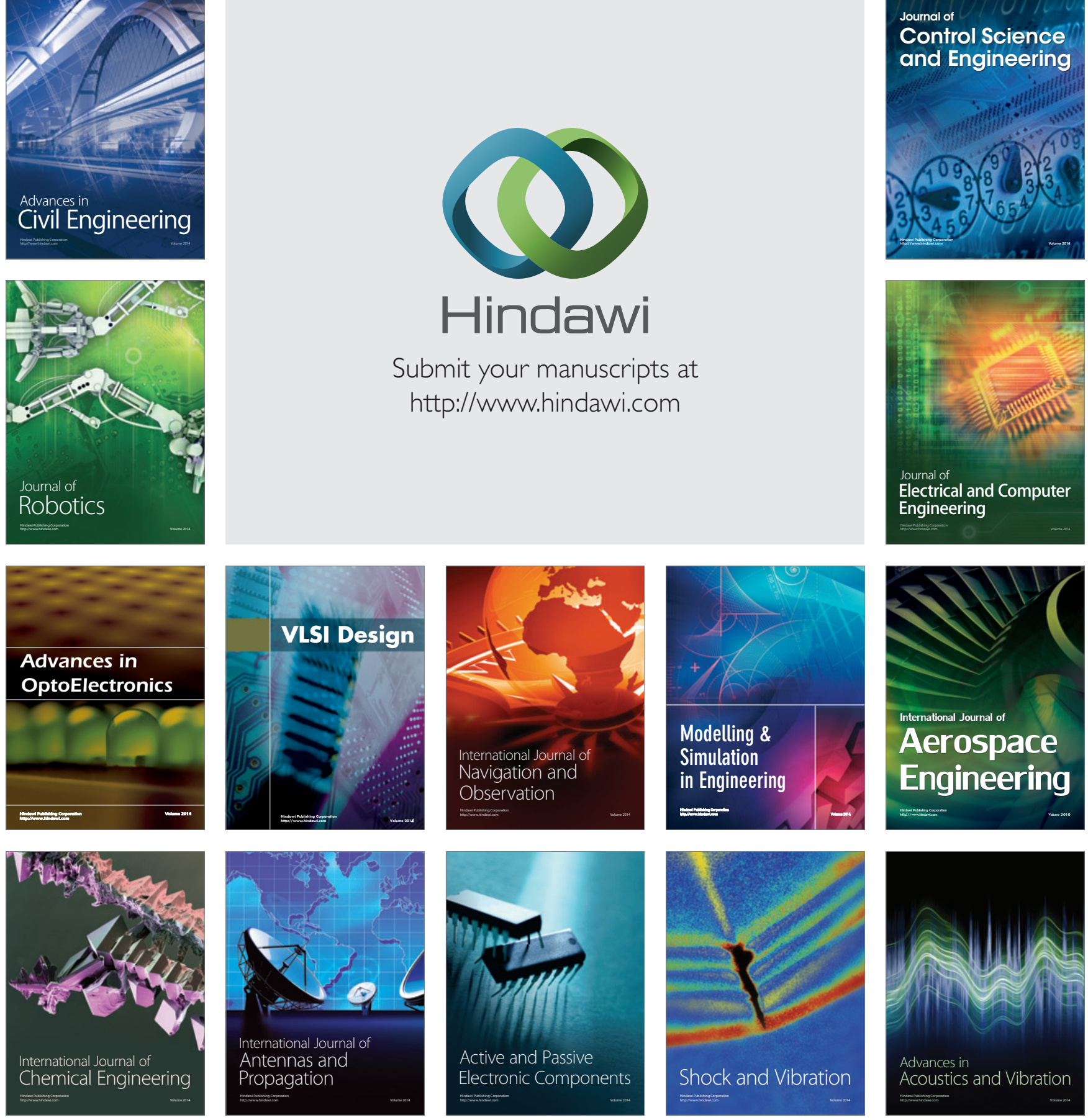